\title{
Iodine Promotes Tumorigenesis of Thyroid Cancer by Suppressing Mir-422a and Up- Regulating MAPK1
}

\author{
Junyi Wang Haiou Yang Yiran Si Dongzhi Hu Yang Yu Yan Zhang \\ Ming Gao Haiyang Zhang \\ Tianjin Medical University Cancer Institute and Hospital, National Clinical Research Center for Cancer, \\ Tianjin Key Laboratory of Cancer Prevention and Therapy, Tianjin's Clinical Research Center for Cancer, \\ Tianjin, China
}

\section{Key Words}

Mir-422a • MAPK1 $・$ Iodine $•$ Thyroid carcinoma $•$ Tumor growth

\begin{abstract}
Background/Aims: Iodine may trigger tumorigenesis and development of thyroid carcinoma, but the mechanisms involved remained elusive. MicroRNA (MiRNAs) are known to be involved in each stage of cancer development; however, the role of miRNAs in iodine-induced tumorigenesis of thyroid carcinoma remained unknown. In this study, we aimed at investigating miRNA related signaling pathway in thyroid cancer cells. Methods: Levels of miRNAs and mRNAs were determined using RT-qPCR and proteins were quantified by western blotting. Cell migration and proliferation were checked using Transwell assay and CCK8 assay respectively. Tumor xenografts in nude mice were established by subcutaneous injection of cancer cells. Results: Mitogen activated protein kinase 1 (MAPK1) was significantly up-regulated, while miR-422a was down-regulated in thyroid cancer cells cultured with high iodine; miR-422a directly bound to the $3^{\prime} U T R$ of MAPK1 mRNA. Moreover, miR-422a negatively regulated MAPK1 expression, and down-regulated miR-422a promoted proliferation and migration of TPC-1 cells. In vivo studies also confirmed that iodine promoted tumor growth by suppressing miR-422a and up-regulating MAPK1. Conclusions: Our study illustrates a new pathway comprising iodine, miRNA and MAPK1, and defines a novel mechanism in thyroid cancer.
\end{abstract}

\section{Introduction}

The incidence of thyroid carcinoma (TC) is the highest among the endocrine malignancy [1]. In addition to follicular TC, the vast majority of TC originates from follicular epithelial cells [2]. The approaches for diagnosis and therapy have greatly improved, and a significant proportion of patients were well treated by surgical operation followed by adjuvant radioactive iodine, but a fraction of patients do not respond to the treatment. Previous 
studies have shown that many genetic and environmental factors induce tumorigenesis and progression, such as radiation exposure [3, 4], estrogen [5] and iodine level [6].

Iodine is raw material for the synthesis of thyroid hormone [7], and is associated with thyroid disease especially TC[8, 9]. Iodine maintains the stability of the thyroid cell internal environment, but it also induces tumorigenesis and progression. In this study, we investigated the internal changes at molecular level in TC cells with high iodine. Better understanding of the molecular mechanisms would contribute to improve diagnosis, therapy and prevention.

Mitogen-activated protein kinase (MAPK) is a kind of protein kinases that acts on serine, threonine, and tyrosine [10]. MAPKs belong to the CMGC (CDK/MAPK/GSK3/CLK) kinase group [11]. Cellular directing responses to a diverse array of stimuli, such as mitogens, osmotic stress, directing heat shock and proinflammatory cytokines is associated with MAPKs. Various of cell functions including proliferation [12], gene expression, cell differentiation, mitosis [13], cell survive, and apoptosis are regulated by MAPKs [14, 15] .

MicroRNAs (miRNAs) are a series of short (18-24 nucleotides in length), endogenous, single-stranded, highly conserved noncoding RNAs which bind to the $3^{\prime}$-translated region $\left(3^{\prime}\right.$ UTR) of target mRNAs to regulate gene expression at the posttranscriptional level via mRNA cleavage or translation inhibition. Previous studies have showed that miRNAs involved in numerous biological processes, including the cell proliferation, cell cycle, apoptosis, migration, invasion, and differentiation [16-20]. MiRNAs have been reported to play a crucial role in development and progression of cancer, and act as critical regulators to in a variety of malignant tumors, such as breast cancer, gastric cancer, colorectal cancer, hepatocellular carcinoma, bladder cancer, renal cell carcinoma and non-small cell lung cancer.

MiR-422a has been identified as a regulator in colorectal cancer, head and neck squamous cell carcinoma, osteosarcoma [21], glioblastoma [22], and hepatocellular carcinoma [23]. However, the role of miR-422a in TC has not been investigated yet. In the present study, we found that miR-422a is suppressed, whereas MAPK1 expression is significantly up-regulated in TC with high iodine. MiR-422a can directly bind with the 3 'UTR of the mRNA of MAPK1 that detected by luciferase assay. The inhibition of miR-422a in TC cells causes the overexpression of MAPK1, and promoted cell proliferation and migration. High consumption of iodine is also confirmed to promotes tumor growth by regulating miR-422a-MAPK1 pathway. Consequently, our data demonstrate the tumorigenesis of TC is closely related with the miR-422a-MAPK1 pathway, which might serve for clinical use in the future.

\section{Materials and Methods}

\section{Cell culture}

The human papillary thyroid carcinoma cell lines TPC-1, K1, and human thyroid cell line Nthy 3, were cultured in RPIM1640 (Gibco, USA) or DMEM (Ghibco, USA) contained 10\% fetal bovine serum and $1 \%$ streptomycin-penicillin, and maintained in a humidified incubator at $37^{\circ} \mathrm{C}$ with $5 \% \mathrm{CO}$.

The miRNA target prediction and Luciferase reporter assay

The prediction and analysis of miRNA target was adopted the algorithms from TargetScan (http:// www.targetscan.org/), PicTar (http://pictar.mdc-berlin.de/) and miRanda (http://www.microrna.org/). Part of the wild type and mutated 3'UTR of MAPK1 which was cloned immediately downstream of the firefly luciferase reporter was inserted into the p-MIR-MAPK1. For the subsequent luciferase reporter assays, $2 \mathrm{mg}$ of firefly luciferase reporter plasmid, $2 \mathrm{mg}$ of $\beta$-galactosidase vector, and equal doses ( $200 \mathrm{pmol})$ of mimics, inhibitors, or scrambled negative control RNA were transfected into the prepared cells. And the transfection control adopted $\beta$-galactosidase vector. At $24 \mathrm{~h}$ after transfection, cells were validated using the Luciferase Assay Kit.

Cell transfection

TPC-1 cells and K1 cells were seeded in six-well or other plates and transfection performed after 24 hours, respectively. For miRNA overexpression and downregulation, added equal amounts of (100 pmol) 


\section{Cellular Physiology Cell Physiol Biochem 2017;43:1325-1336 \begin{tabular}{l|l|l} 
and BOI: 10.1159/000481844 & $\begin{array}{l}\text { C) } 2017 \text { The Author(s). Published by S. Karger AG, Basel } \\
\text { www.karger.com/cpb }\end{array}$
\end{tabular}}

Wang et al.: Iodine-Mir-422a Regulates Tumorigenesis of TC

scrambled negative control, miR-422a mimics and inhibitors with lipofectamine 2000 (Invitrogen, Life Technologies) and Opti-MEM Reduced Serum Medium (Gibco, Life Technologies) into each well according to the manufacturer's instructions.

Then after $24 \mathrm{~h}$, the transfected cells were harvested, for real-time quantitative PCR analysis and at $48 \mathrm{~h}$ for western blotting.

\section{RNA isolation and quantitative RT-PCR}

Total RNA was extracted from the cultured cells, using TRIzol Reagent (Invitrogen) according the manufacturer's protocol. The quantity of miRNA was assayed via Taqman microRNA probes (Applied Biosystems, Foster City, CA). All of the reactions were run in triplicate, after the reactions were completed, the cycle threshold (CT) data were determined using fixed threshold settings, and the mean CT was calculated from triplicate PCRs. A comparative CT method was adopted to compare each condition to the control reactions. U6 snRNA was used as an internal control of miRNAs, and the mRNA levels of MAPK1 was normalized to the corresponding housekeeping gene GAPDH. The relative amount of gene normalized to control was calculated with the equation 2- $\Delta \mathrm{CT}$, in which $\Delta \mathrm{CT}=\mathrm{CT}$ gene $-\mathrm{CT}$ control. Primers of MAPK1, NIS and GAPDH were as follows:

5'- ACGCCAAACGCATCTACAG-3' (MAPK1 sense);

5'- AACGAAAGACCTAAGAGCAGC-3' (MAPK antisense);

5'-TCTCTCAGTCAACGCCTCT-3' (NIS sense);

5'-ATCCAGGATGGCCACTTCTT-3' (NIS antisense);

5'-AGAAGGCTGGGGCTCATTTG-3' (GAPDH sense);

5'-AGGGGCCATCCACAGTCTTC-3' (GAPDH antisense).

\section{Western blotting}

The level of MAPK1 expression was measured by western blotting analysis and samples were normalized to GAPDH. Total protein was extracted from the cultured cells, and it was lysed in lysis buffer. The protein were separated by sodium dodecyl sulfate polyacrylamide gel electrophoresis and then transferred to polyvinylidene difluoride membranes (Roche). The membranes were blocked within $2 \%$ Bovine Serum Albumin (BSA) at room temperature for $1 \mathrm{~h}$ and incubated overnight at $4^{\circ} \mathrm{C}$ with primary anti-MAPK1 (1:200, Santa Cruz), and anti-GAPDH (1:5000, Santa Cruz), respectively. The membranes subsequently washed and incubated with corresponding secondary antibodies. After disposed with ECL, the protein bands were visualized with an enhanced chemiluminescence system kit.

\section{Cell proliferation assay}

Cells including TPC-1cells, K1 cells and Nthy-3 cells were collected at 12 h, 24 h, 36 h and 48 h which were cultured in different iodine level or transfected differently ; $10 \mu \mathrm{L}$ of WST-8 was added into a corresponding test well and incubated for $4 \mathrm{~h}$. Absorbance was measured at a wavelength of $450 \mathrm{~nm}$.

\section{Cell migration assay}

Transwell-chamber (Corning, New York, USA) migration assay was adopted to determine the migrative capacity of transfected cells and cells cultured with different iodine level. The Transwell-chamber test divided into two parts, one is for different post-transfection cells , the other is for the cells which were cultured with different concentration of KI. Cells were transferred into the upper chamber of the transwell which contains $200 \mu \mathrm{l}$ serum-free growth medium (105 cells per well of $8.0 \mu \mathrm{m}$ Pore Polycarbonate Membrane Insert)., Complete medium containing $10 \%$ FBS was added to the lower chamber as a chemo-attractant. After $24 \mathrm{~h}$ of incubation at $37{ }^{\circ} \mathrm{C}$, nonmigratory cells on the upper surface of upper chamber were slightly removed by cotton swabs, and cells migrated to the bottom of the membrane were fixed and stained. The number of invaded cells was counted under light microscope. To minimize the bias, five randomly selected fields with $200 \times$ magnification were counted, then the average number was calculated.

\section{Establishment of tumor xenografts in nude mice}

TPC-1 cells were injected subcutaneously into nude mice $\left(1 \times 10^{7}\right.$ cells per mouse). Twenty micrograms of cell-derived MVs were injected per mouse via intravenous tail injection every two days. Mice were sacrificed after 4 weeks, and the weights and diameters of tumors were recorded. 


\section{Statistical analyses}

All data are representative of five or six independent experiments. The data were expressed as the mean values \pm S.E. of at least five separate experiments. Statistical significance was considered as $P<0.05$ using Student's t-test. In this study, '*', '**', and ${ }^{* * * *}$ indicate ' $\mathrm{P}<0.05^{\prime}$, ${ }^{\prime} \mathrm{P}<0.01^{\prime}$, and $\mathrm{P}<0.001^{\prime}$, respectively.

\section{Results}

\section{High iodine up-regulates MAPK1 expression in thyroid cancer cells}

In order to detect the relationship between the quantity of MAPK1 cells and iodine in thyroid cancer, expression of MAPK1 was detected by western blotting and qRT-PCR analysis. We analyzed three cells lines, including TPC-1 cells, K1 cells and Nthy-3 cells, which were

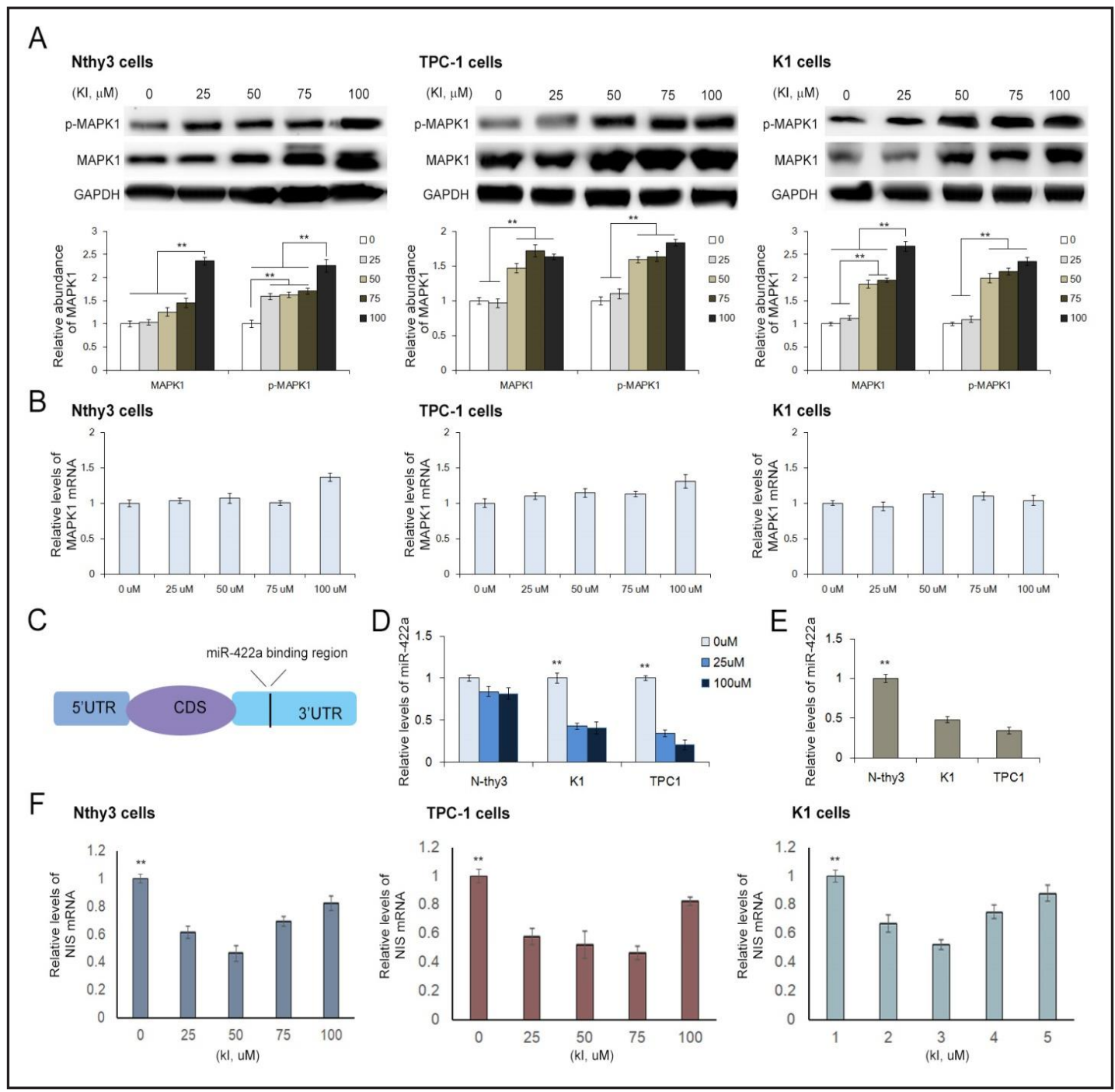

Fig. 1. The expression patterns of MAPK1 and miR-422a in cells.A. Western blot analysis of MAPK1 expression in Nthy-3 cells, TPC-1 cells and K1 cells with different KI concentration ( $n=3)$. B. Relative levels of MAPK1 mRNA levels in Nthy-3 cells, TPC-1 cells and K1 cells $(n=3)$. C. The predicted binding region of miR-422a in the mRNA of MAPK1. D. Relative levels of miR-422a in Nthy-3 cells, TPC-1 cells and K1 cells with different KI concentration (n=3). E. Determination of miR-422a expression in three TC cell lines ( $n=3)$. F. Relative levels of NIS mRNA in three cells lines treated with high iodine $(n=3)$. “**” indicates $p<0.01$.

\section{KARGER}


cultured in five different concentrations of $\mathrm{KI}(0 \mu \mathrm{M}, 25 \mu \mathrm{M}, 50 \mu \mathrm{M}, 75 \mu \mathrm{M}, 100 \mu \mathrm{M})$. As shown in Fig. 1A, MAPK1 protein was found to be significantly upregulated in TPC-1 cells and K1 cells with the increasing concentration of KI, but not in Nthy-3 cells. However, the mRNA level of MAPK showed only slightly increase in these three cells lines (Fig. 1B). These results indicated that cancerous thyroid cells are much more sensitive to iodine than normal thyroid cells.

\section{MiR-422a is down-regulated in thyroid cancer cells and directly targets MAPK1}

In the posttranscriptional regulatory mechanisms, miRNA-mediated specific mRNA cleavage or translational repression plays a crucial role. In our study, based on the predictive results of bioinformatics tools, we find that miR-422a can directly target the $3^{\prime}$ UTR of MAPK1 mRNA, and the free energy of all three computational algorithms showed that miR422a has high affinity with MAPK1. As shown in Fig. 1C and Fig. 2A, the position that miR422a directly binds with MAPK1 mRNA. To confirm the actual relationship of miR-422a and MAPK1 in thyroid cancer, we explored the level of miR-422a in TC cells with KI concentration of $(0 \mu \mathrm{M}, 25 \mu \mathrm{M}, 100 \mu \mathrm{M}$, respectively). As is expected, miR-422a showed obvious decrease in TC cells $(\mathrm{p}<0.01$ ) (Fig. 1D). The levels of miR-422a and MAPK1 showed inverse correlation in TC with high iodine level. The differences of miR-422a expression in three cell lines were also determined. The miR-422a level was much higher in N-thy3 cells compared with other two cancer cell lines (Fig. 1E). NIS mRNA was inhibited by moderate doses of iodine, while the inhibition was decreased by high levels of iodine (Fig. 1F). Therefore, in high iodine environment; miR-422a is most likely to be the important regulator of MAPK1 in thyroid

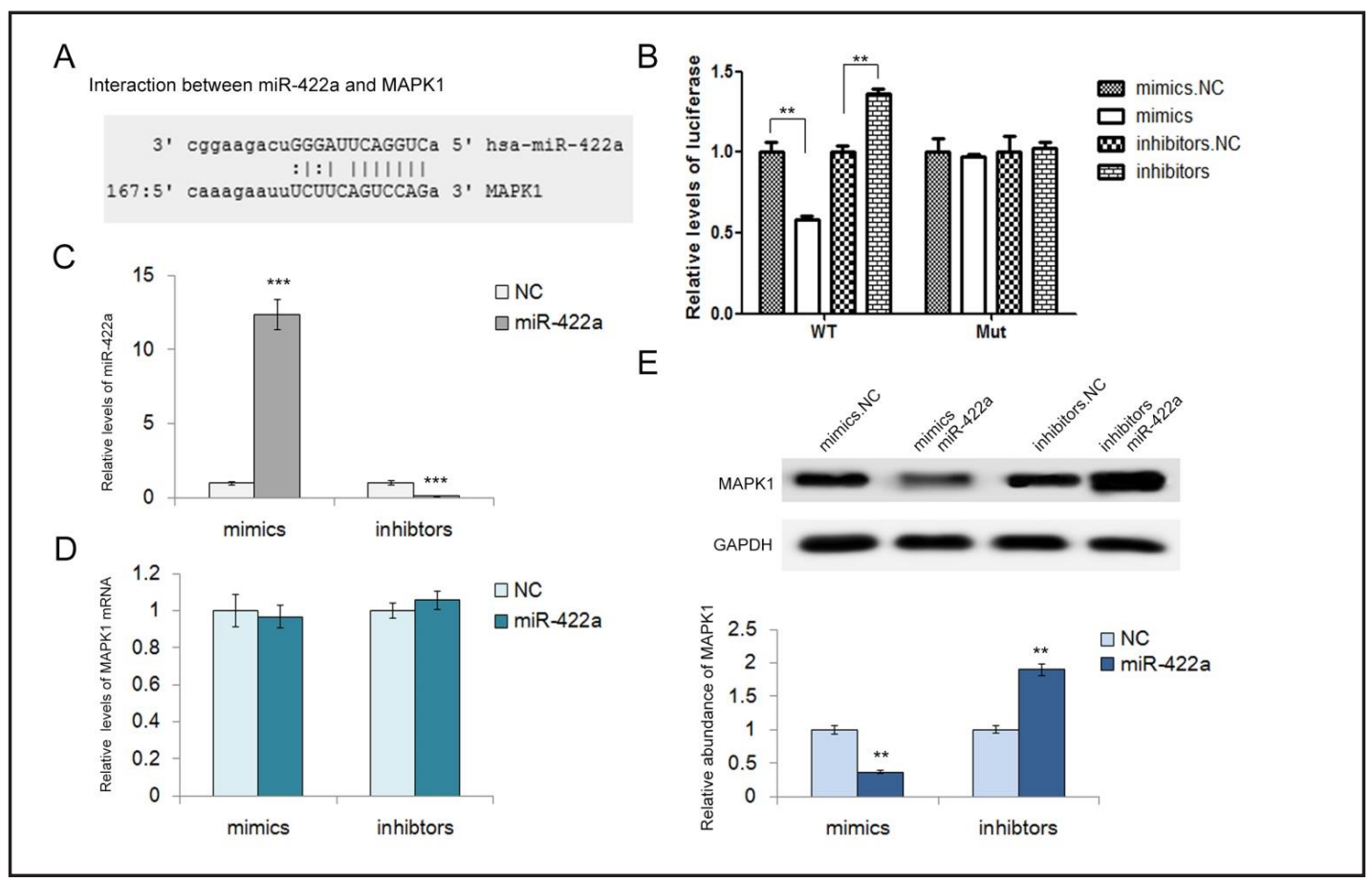

Fig. 2. MiR-422a suppresses MAPK1 expression in thyroid cancer cells. A. The predicted base-pairing interaction between miR-422a and MAPK1 mRNA. B. Direct recognition of MAPK1 by miR-422a. TPC-1 cells were co-transfected with firefly luciferase reporters containing either WT or mutant MAPK1 3'UTR with miR-422a mimics and inhibitors ( $n=3)$. C. Quantitative RT-PCR analysis of miR-422a levels in TPC- 1 cells transfected with mimics or inhibitors ( $n=3$ ). D. Quantitative RT-PCR analysis of MAPK1 mRNA levels in TPC1 cells transfected with miR-422a mimics or inhibitors $(n=3)$. E. Western blot analysis of MAPK1 expression in TPC-1 cells transfected with miR-422a mimics or inhibitors $(\mathrm{n}=3)$. “**” indicates $\mathrm{p}<0.01$; “***” indicates $\mathrm{p}<0.001$.

\section{KARGER}


cancer cells. Then we performed luciferase assay to investigate the direct interaction between miR-422a and MAPK1. In order to verify this targeting relationship, we inserted the miR-422a binding sequence in the 3'-UTR of MAPK1 and the mutated 3'-UTR sequence into the downstream of the firefly luciferase reporter gene in p-MIR vector, and then it was co-transfected with miR-422a mimics, inhibitors (or miRNA NC) into TPC-1 cells. As shown in Fig. 2B, the relative luciferase activity of the reporter gene in TPC-1 cells which were cotransfected with p-MIR-MAPKand miR-422a mimics showed significantly decreased by nearly $50 \%$ compared with the control (co-transfected with p-MIR-MAPK1 and miRNA NC), while the relative luciferase activity of the reporter gene in TPC-1 cells that were co-transfected with mutated p-MIR-TGF $\beta R 2$ and miR-422a mimics or miRNA NC had no significant changes. While TPC-1 cells were transfected with miR-422a inhibitors revealed that the luciferase signal increased. Our results verified that miR-422a could suppress TGF $\beta$ R2 expression via binding to the 3'UTR of MAPK1 mRNA.

\section{MiR-422a suppresses MAPK expression in TPC-1 cells}

In order to demonstrate the function of miR-422a, we transfected miR-422a mimics or inhibitors into TPC-1 cell line. After incubated $24 \mathrm{~h}$, the cells were collected for detecting miR-422a levels by qRT-PCR analysis. Results showed that miR-422a mimics could significantly increase miR-422a level in TPC-1 cells, while miR-422a inhibitors decreased miR-422a level (Fig. 2C). To further investigate whether miR-422a regulated expression at post-transcriptional level in TC cells, we adopted qRT-PCR and Western blot to detect the mRNA and protein level of MAPK1 in TPC- 1 cells that were transfected by miR-422a mimics or inhibitors. As shown in Fig. 2D and 2E, the overexpression or lower expression of miR422a by transfection of mimics or inhibitors did not lead to different mRNA expression of

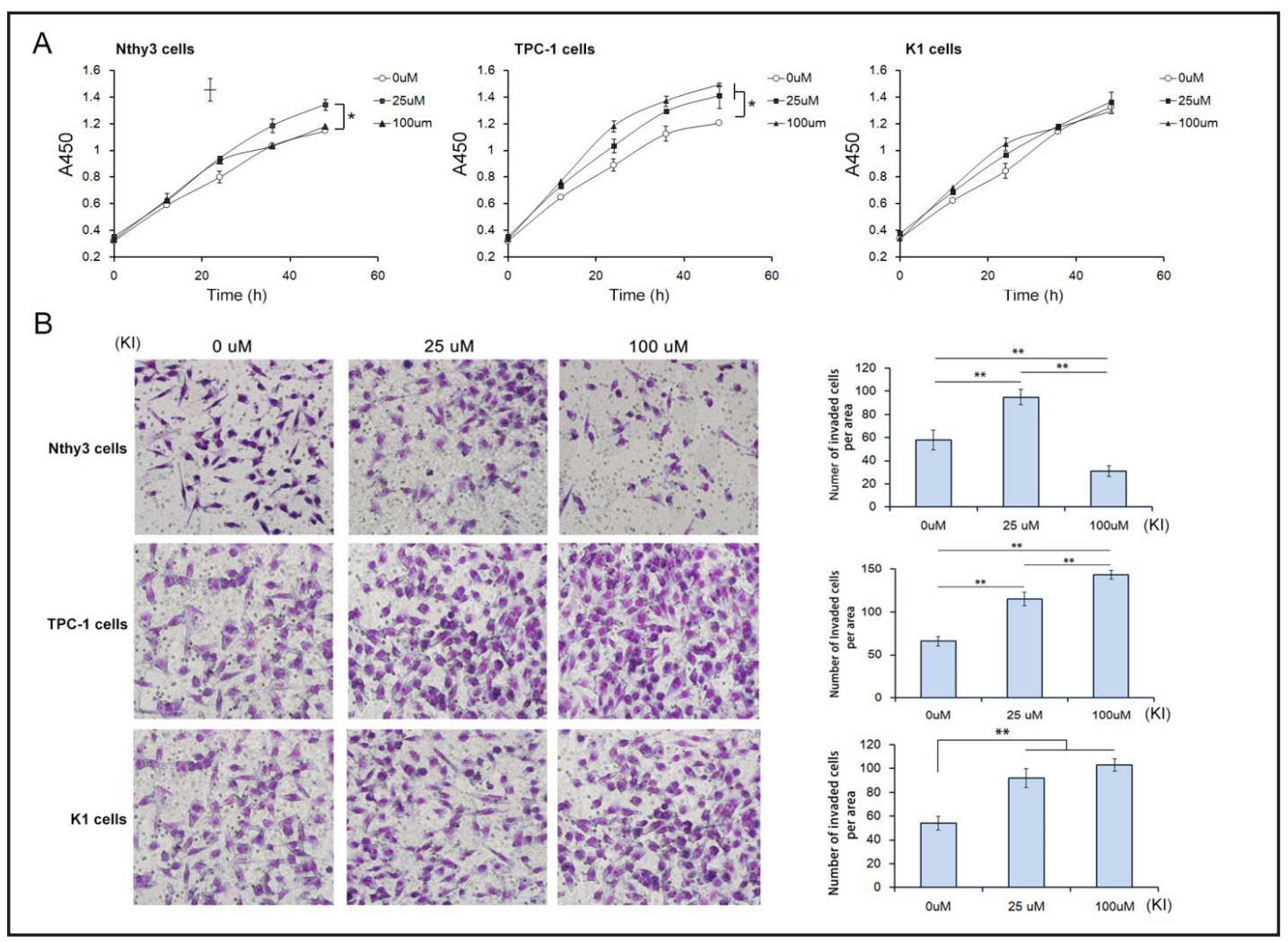

Fig. 3. Role of Iodine in thyroid cancer cells. A.CCK- 8 kit for analyzing the proliferation of TPC-1 cells, K1 cells and Nthy-3 cells with different KI concentrates ( $n=3)$. B. Transwell-chamber migration assay for the exploring migration of TPC- 1 cells line, K1 cells line and Nthy-3 cells line with different KI concentrates $(n=3)$. “*” indicates $\mathrm{p}<0.05$; “**” indicates $\mathrm{p}<0.01$. 
MAPK1. But the up-regulated of miR-422a by transfected with miR-422a mimics induced a significant lower expression of MAPK1 protein. These data was a further evidence that miR$422 \mathrm{a}$ is an important post-transcriptional regulator of MAPK1 in TC cells.

\section{Iodine regulates proliferation and migration of TPC-1 cells}

For investigating the effects of iodine levels on thyroid cancer cells, we performed CCK-8 kit for analyzing the proliferation and Transwell-chamber migration assay for the exploring migration of TPC-1 cells line, K1 cells line and Nthy-3 cells line with different KI concentrates $(0 \mu \mathrm{M}, 25 \mu \mathrm{M}, 100 \mu \mathrm{M})$. The result showed that TPC- 1 cells were more sensitive to the improvement of KI's concentrate, and the capability of proliferation (Fig. 3A) and migration (Fig. 3B) was significantly promoted. And the proliferation and migration of TPC1 cells with $100 \mu \mathrm{M}$ KI increased more obvious than other two cell lines with $25 \mu \mathrm{M} \mathrm{KI}$ $(\mathrm{p}<0.01)$. However, the proliferation and migration of K1 cells and Nthy-3 cells were almost not changed, except Nthy-3 cell line's proliferation with $100 \mu \mathrm{M}$ KI had a slightly increase and its migration with $100 \mu \mathrm{M} \mathrm{KI}$ was repressed.

\section{MiR-422 a regulates cell proliferation and migration in TPC-1 cells}

MiR-422a serves as a regulator promotes the proliferation and migration of TPC- 1 cells. In vitro, we adopted CCK-8 kit to analyses the effect of miR-422a on the proliferation of TPC1 cells. The results revealed that the proliferation rate in TPC- 1 cells transfected with miR422a mimics was significantly decreased compared with the control group (Fig. 4A). On

Fig. 4. MiR-422a regulates cell proliferation and migration in TPC-1 cellsA. CCK-8 kit for analyzing the proliferation of TPC-1 cells transfected with miR-422a mimics or inhibitors $(n=3)$. B. CCK-8 kit for analyzing the proliferation of N-thy3 cells transfected with miR-422a mimics or inhibitors $(n=3)$. C. Transwell-chamber migration assay for the exploring migration of TPC-1 cells transfected with miR-422a mimics or inhibitors $(n=3)$. D. Transwell-chamber migration assay for the exploring migration of $\mathrm{N}$-thy3 cells transfected with miR-422a mimics or inhibitors. "**" indicates $\mathrm{p}<0.01$.




the contrary, the proliferation rate in TPC-1 cells transfected with miR-422a inhibitors increased than the NC inhibitor group (Fig. 4A). Transwell-chamber migration assay was used to measure the migration ability changes of TPC-1 cells. As shown in Fig. 4C, overexpression of miR-422a suppressed the migration of TPC-1 cells. Inversely, the migration rate of TPC-1 cells which were transfected by miR422a inhibitor was increased. However, the biological behavior of $\mathrm{N}$-thy 3 cells showed little change by the transfection of miR-422a mimics or inhibitors (Fig. 4B and 4D).

\section{Silencing of miR-422a in TPC-1 cells}

To validate that iodine promotes MAPK1 expression via inhibiting miR-422a, we knock out miR-422a in TPC- 1 cells by using a lenti-virus containing miR-422a shRNA. As is shown in Fig. 5A, miR-422a was significantly down-regulated in TPC-1 cells, while enhancing the expression level of MAPK1 (Fig. 5B). As is expected, addition of KI cannot lead to a higher level of MAPK1 with absence of miR-422a(Fig. 5C and 5D). Moreover, high level of iodine has little effects on cell proliferation and migration in miR-422a-KO cells (Fig. 5E and 5F).

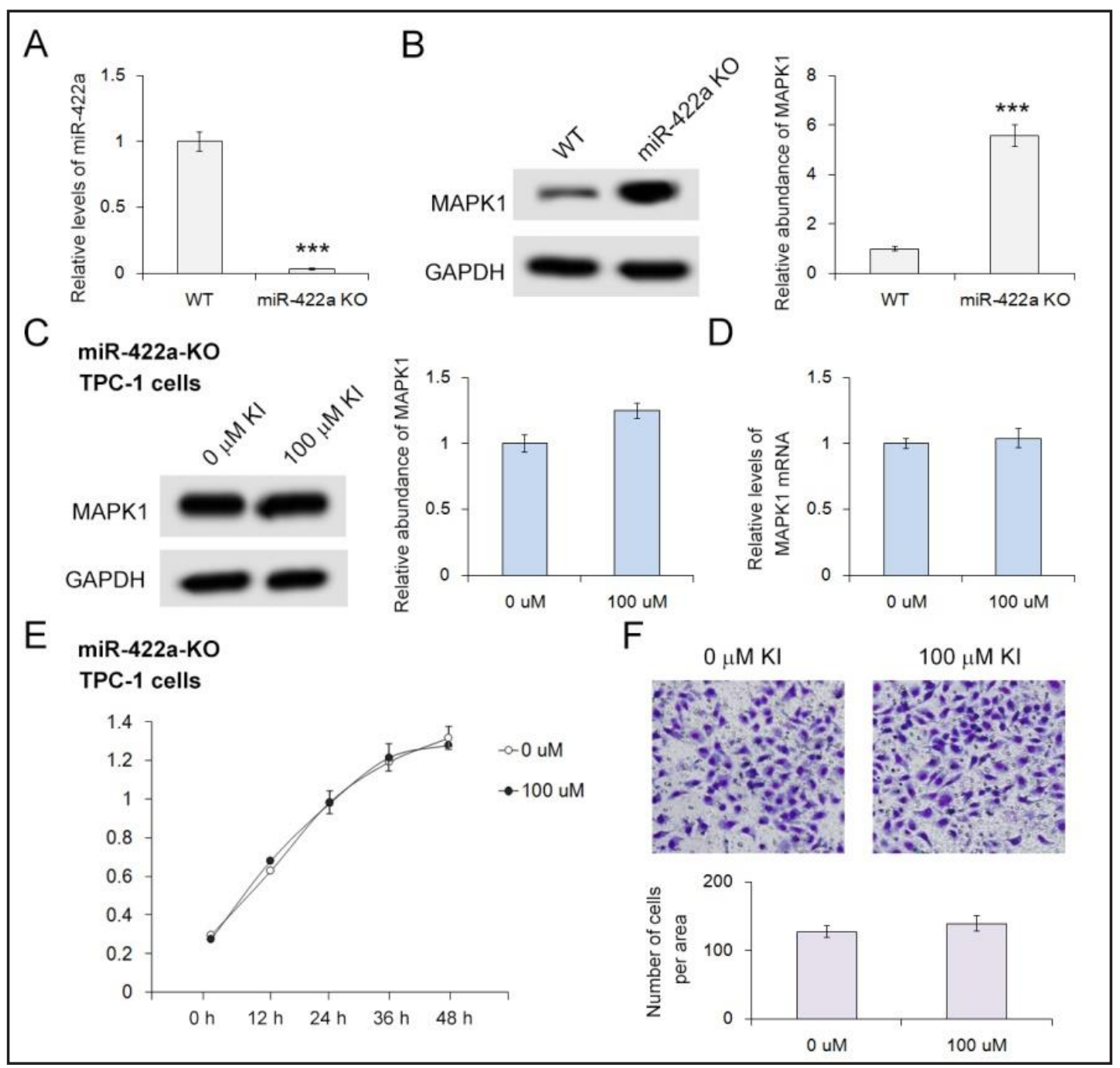

Fig. 5. Knock-down of miR-422a in TPC-1 cells. A. Relative levels of miR-422a in WT and miR-422a-KO TPC1 cells $(n=3)$. B. Western blot analysis of MAPK1 expression in miR-422a-abscent cells. miR-422a-abscent TPC-1 cells were treated with 100 m Iodine, and expression of MAPK1, cell growth and migration were checked ( $\mathrm{n}=3$ ). Levels of MAPK1 protein (C) and mRNA (D) in miR-422a-KO TPC-1 cells treated with KI. Cell proliferation (E) and migration (F) of miR-422a-KO TPC-1 cells $(\mathrm{n}=3)$. “***” indicates $\mathrm{p}<0.001$. 


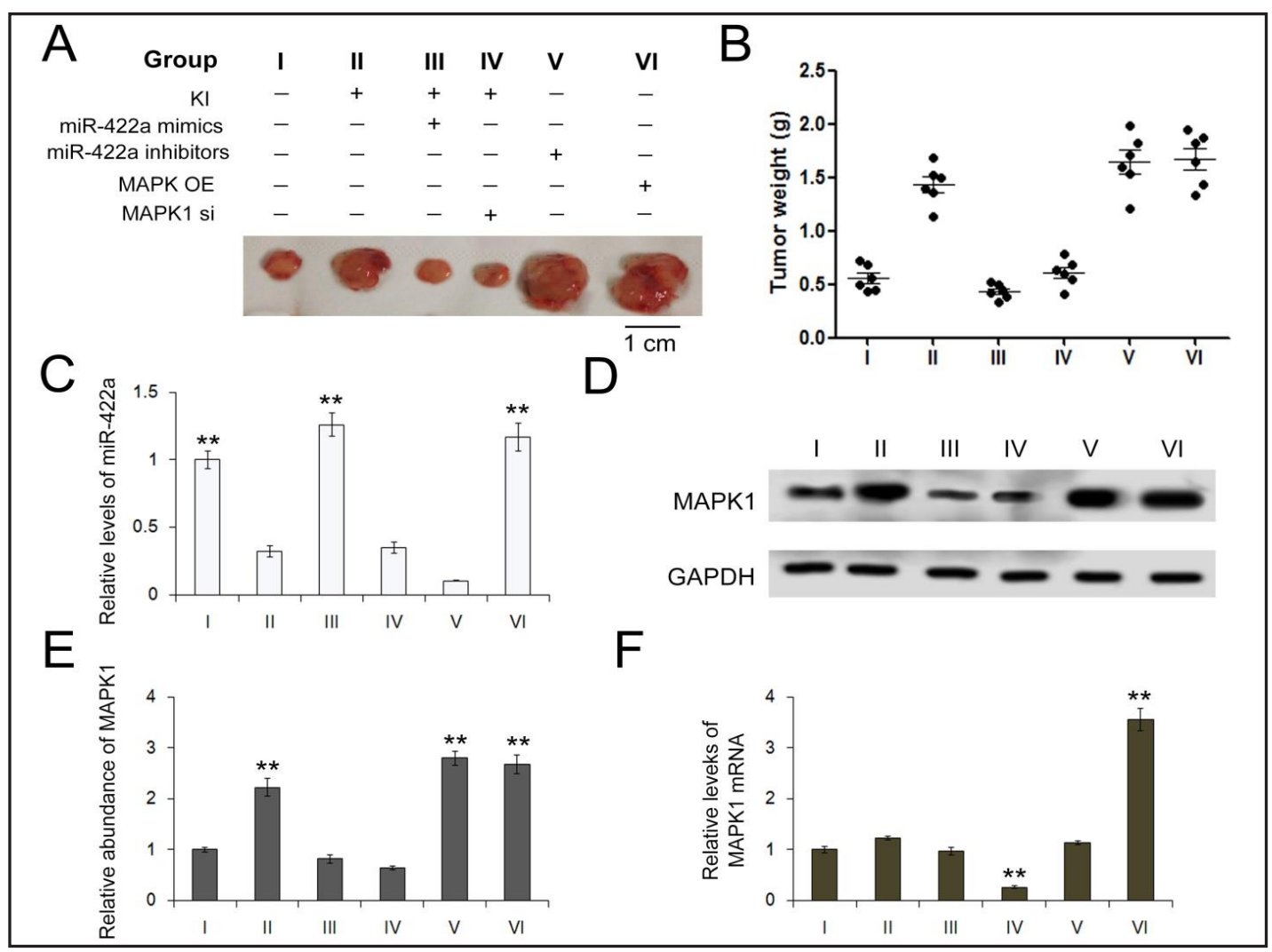

Fig. 6. In vivo role of iodine/miR-422a/MAPK1 in thyroid cancer. A. Images of thyroid tumors in mice $(n=6)$. B. The weight of tumors of A ( $n=6)$. C. Relative levels of miR-422a in tumors of each group $(n=6)$. D. Western blot analysis of MAPK1 in tumors $(n=6)$. E. Quantitative analysis of D $(n=6)$. F. RT-qPCR analysis of MAPK1

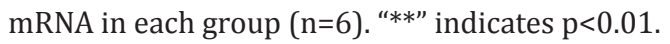

These results further demonstrated the important role of miR-422a in iodine-induced tumorigenesis of thyroid cancer.

In vivo role of miR-422a in TC treated with high iodine

High levels of iodine is believed to be closely linked with the development of thyroid cancer [24].We evaluated the effects of iodine on tumor growth and the expression of miR422a/MAPK1 using tumor implanted mouse model. Mice were feed with water containing $50 \mu \mathrm{M} \mathrm{KI}$ to get high consumption of iodine. It was shown that high iodine accelerates tumor growth in vivo; while overexpressed miR-422a, as well as down-regulated MAPK1 significantly suppress the growth of thyroid tumor (Fig. 6A and 6B). As is expected, high iodine down-regulates the expression of miR-422a and activates MAPK1 pathway at the transcriptional level (Fig. 6C, 6D, 6E and 6F). The in vivo results were consistent with the data obtained from cell experiments, and implied that iodine may serve as a positive factor in TC.

\section{Discussion}

Most of thyroid carcinoma occurs in coastal areas, previous studies have clearly indicated that both Iodine deficiency and iodine excess have impact on the tumorigenesis and progress of TC [25-27], and the mechanisms underlying these two conditions are different for TC. Iodine deficiency inducing thyroid disorders has been clearly defined [28]. However the impact of iodine excess on TC is not explicitly known. It is meaningful to explore 


\section{Cellular Physiology Cell Physiol Biochem 2017;43:1325-1336 \\ \begin{tabular}{ll|l} 
DOI: 10.1159/000481844 & O 2017 The Author(s). Published by S. Karger AG, Basel \\
www.karger.com/cpb
\end{tabular} \\ Wang et al.: Iodine-Mir-422a Regulates Tumorigenesis of TC}

the influence of iodine excess on the TC cells.

MicroRNAs mediate a variety of physiological and pathological processes in the cell through targeting gene and regulating relative protein expression. Compared with the normal thyroid cells, the MAPK1 protein was increased in TC cells with high iodine, while miR-422a expression level was dramatically repressed in TC cells. This change was closely related with iodine concentration. With the increase of iodine concentration in TPC-1 cells, the change was even greater. MAPK1 protein expression was inversely correlated with miR422a in TC cells with high iodine level, but the MAPK1 mRNA showed little increase in TC cells. However, K1 cells line has B-Raf mutation with a hyper-activation of MAPK. In higher iodine concentration $(100 \mu \mathrm{M})$ the migration ability of K1 cells was promoted. Correlatively, down-regulated miR-422a also enhanced the proliferation and migration of TPC-1 cells.

Our data verified that iodine excess regulates the biological behavior of TC cells via miR422a/MAPK1 pathway. In addition, we found that the expression of MAPK1 had a significant increase in Nthy-3 cells with high iodine concentration $(100 \mu \mathrm{M})$. It showed that high iodine environment may induce the dys-regulation of miR-422a-MAPK1 pathway in normal thyroid cells. It may be a potential molecular mechanism of thyroid carcinoma that induced by high iodine. However, this needs more evidence in further studies, since we performed the function experiments in only two iodine concentrations.

In most individuals, escape from the Wolff-Chaikoff effect caused by acute excess occurs due to a decrease in sodium-iodide symporter (NIS) activity. NIS mRNA was relatively downregulated by high iodine, implying that escape from Wolff-Chaikoff effect may contribute to the regulation of cellular biological behavior. But MAPK1 pathway is important in regulating a variety of cellular activities and many life process involved in many human diseases including tumor development, the role of MAPK1 signaling pathway in tumorigenesis is widely confirmed.

The highly conserved tissue miRNAs could provide an accurate diagnosis for various types of malignancies, which usually lead to disorders of protein expression in cancer cells. For all we know, this is the first attempt to explore the miR-422a-MAPK1 pathway in TC cells with different iodine concentration. Our results show that miR-422a act as a potential tumor suppressor in GC. Currently, surgical followed by adjuvant radioactive iodine is the major viable strategies exist for the treatment of TC. But a fraction of patient has no effective response to this therapy. It drives urgent need to detect new targets and treatment strategies. And our study may be a novel treatment target for the group of TC patients that caused by high iodine. MiRNAs act as a valuable biomarker in the diagnosis and prognosis of cancer involved tumorigenesis, angiogenesis and drug resistance. Nowadays in mouse cancers models, miRNAs or anti-miRNAs trafficked by microvesicles (MV) or other plasmids have been used for the treatment.

\section{Conclusion}

The miR-422a-MAPK1 pathway in high iodine environment is involved in the processes of cell proliferation and migration, thus regulating tumorigenesis in TC. Our study provided further evidence of the effects of iodine on thyroid cancer, and miR-422a might act as a suppressor of TC. Further studies are necessary to investigate its clinical application as potential molecular diagnosis and therapeutic target for human TC.

\section{Acknowledgements}

This work was supported by grants from the National Natural Science Foundation of China (Nos. 81602158). This work was also supported by Doctoral foundation of Tianjin Medical University Cancer Institute and Hospital (B1210). The funders had no role in study design; collection, analysis, and interpretation of data; in the writing of the report; and in the decision to submit this article for publication. 


\section{Cellular Physiology Cell Physiol Biochem 2017;43:1325-1336

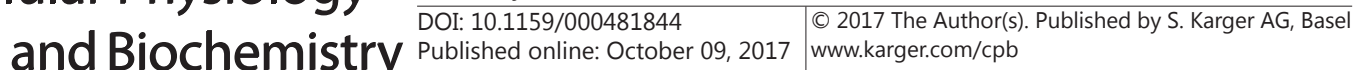

Wang et al.: Iodine-Mir-422a Regulates Tumorigenesis of TC

\section{Disclosure Statement} article.

The authors declare that there is no conflict of interests regarding the publication of this

\section{References}

1 Hu Y, Wang H, Chen E, Xu Z, Chen B, Lu G: Candidate microRNAs as biomarkers of thyroid carcinoma: a systematic review, meta-analysis, and experimental validation. Cancer Med 2016;5:2602-2614.

-2 Pallante P, Visone R, Croce CM, Fusco A: Deregulation of microRNA expression in follicular-cell-derived human thyroid carcinomas. Endocr Relat Cancer 2010;17:F91-104.

3 Greenspan FS: Radiation exposure and thyroid cancer. Jama 1977; 237:2089-2091.

4 Cannizzaro MA, Veroux M, Costanzo M, Buffone A, Okatyeva V: [Radiation exposure and thyroid cancer]. Ann Ital Chir 2012;83:369-372.

5 Derwahl M, Nicula D: Estrogen and its role in thyroid cancer. Endocr Relat Cancer 2014;21:T273-283.

6 Franceschi S: Iodine intake and thyroid carcinoma--a potential risk factor. Exp Clin Endocrinol Diabetes 1998;106:S38-44.

7 Schlienger JL, Goichot B, Grunenberger F: [Iodine and thyroid function]. Rev Med Interne 1997;18:709-716.

8 Woeber KA: Iodine and thyroid disease. Med Clin North Am 1991;75:169-178.

-9 Foley TP, Jr.: The relationship between autoimmune thyroid disease and iodine intake: a review. Endokrynol Pol 1992;43:S53-69.

10 Coulombe P, Meloche S: Atypical mitogen-activated protein kinases: structure, regulation and functions. Biochim Biophys Acta 2007;1773:1376-1387.

11 Rauch N, Rukhlenko OS, Kolch W, Kholodenko BN: MAPK kinase signalling dynamics regulate cell fate decisions and drug resistance. Curr Opin Struct Biol 2016;41:151-158.

12 Zhang W, Liu HT: MAPK signal pathways in the regulation of cell proliferation in mammalian cells. Cell Res 2002;12:9-18.

$\checkmark 13$ Tae JC, Kim EY, Jeon K, Lee KS, Lee CH, Kim YO, Park SP, Kim NH: A MAPK pathway is involved in the control of cortical granule reaction and mitosis during bovine fertilization. Mol Reprod Dev 2008;75:1300-1306.

14 Scuteri A, Galimberti A, Maggioni D, Ravasi M, Pasini S, Nicolini G, Bossi M, Miloso M, Cavaletti G, Tredici G: Role of MAPKs in platinum-induced neuronal apoptosis. Neurotoxicology 2009;30:312-319.

15 Zhu W, Zou Y, Aikawa R, Harada K, Kudoh S, Uozumi H, Hayashi D, Gu Y, Yamazaki T, Nagai R, Yazaki Y, Komuro I: MAPK superfamily plays an important role in daunomycin-induced apoptosis of cardiac myocytes. Circulation 1999;100:2100-2107.

16 Fan C, Wang W, Jin J, Yu Z, Xin XP: RASSF10 is Epigenetically Inactivated and Suppresses Cell Proliferation and Induces Cell Apoptosis by Activating the p53 Signalling Pathway in Papillary Thyroid Carcinoma Cancer. Cell Physiol Biochem 2017;41:1229-1239.

17 Qiu ZL, Shen CT, Sun ZK, Wei WJ, Zhang XY, Song HJ, Luo QY: Circulating Long Non-Coding RNAs Act as Biomarkers for Predicting I-131 uptake and Mortality in Papillary Thyroid Cancer Patients with Lung Metastases. Cell Physiol Biochem 2016;40:1377-1390.

-18 Yan L, Li QH, Li XY, Ji H, Zhang LL: Association Studies Between XRCC1, XRCC2, XRCC3 Polymorphisms and Differentiated Thyroid Carcinoma. Cell Physiol Biochem 2016;38:1075-1084.

19 Wang G, Cai CZ, Chen L: MicroRNA-3666 Regulates Thyroid Carcinoma Cell Proliferation via MET. Cell Physiol Biochem 2016;38:1030-1039.

20 Deng XZ, Wu B, Xiao K, Kang J, Xie J, Zhang XP, Fan YB: MiR-146b-5p Promotes Metastasis and Induces Epithelial-Mesenchymal Transition in Thyroid Cancer by Targeting ZNRF3. Cell Physiol Biochem 2015;35:71-82.

21 Bahador R, Taheriazam A, Mirghasemi A, Torkaman A, Shakeri M, Yahaghi E, Goudarzi PK: Tissue expression levels of miR-29b and miR-422a in children, adolescents, and young adults' age groups and their association with prediction of poor prognosis in human osteosarcoma. Tumour Biol 2016;37:30913095. 


\section{Cellular Physiology Cell Physiol Biochem 2017;43:1325-1336

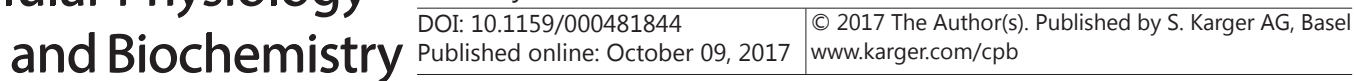

Wang et al.: Iodine-Mir-422a Regulates Tumorigenesis of TC

22 Liang H, Wang R, Jin Y, Li J, Zhang S: MiR-422a acts as a tumor suppressor in glioblastoma by targeting PIK3CA. Am J Cancer Res 2016;6:1695-1707.

23 Zhang J, Yang Y, Yang T, Yuan S, Wang R, Pan Z, Yang Y, Huang G, Gu F, Jiang B, Lin C, Zhou W: Doublenegative feedback loop between microRNA-422a and forkhead box (FOX)G1/Q1/E1 regulates hepatocellular carcinoma tumor growth and metastasis. Hepatology 2015;61:561-573.

24 Ibrahim EY, Busaidy NL: Treatment and surveillance of advanced, metastatic iodine-resistant differentiated thyroid cancer. Curr Opin Oncol 2017;29:151-158.

-25 Liu XH, Chen GG, Vlantis AC, van Hasselt CA: Iodine mediated mechanisms and thyroid carcinoma. Crit Rev Clin Lab Sci 2009;46:302-318.

-26 Kanno J, Onodera H, Furuta K, Maekawa A, Kasuga T, Hayashi Y: Tumor-promoting effects of both iodine deficiency and iodine excess in the rat thyroid. Toxicol Pathol 1992;20:226-235.

27 Du Y, Gao Y, Meng F, Liu S, Fan Z, Wu J, Sun D: Iodine deficiency and excess coexist in china and induce thyroid dysfunction and disease: a cross-sectional study. PLoS One 2014;9:e111937.

-28 Zimmermann MB, Boelaert K: Iodine deficiency and thyroid disorders. Lancet Diabetes Endocrinol 2015;3:286-295. 